\title{
Aceptación de la de vacunación contra la COVID-19 en mujeres peruanas embarazadas: Actitudes y factores asociados
}

\section{Acceptance of COVID-19 vaccination among pregnant Peruvian women: Attitudes and associated factors}

Recibido: 22/04/2021

https://doi.org/10.52808/bmsa.7e5.61e2.005

Daniella Vinelli-Arzubiaga ${ }^{1}$

https://orcid.org/0000-0002-8793-2446

Amparo W. Marquez-Bravo 2

https://orcid.org/0000-0002-4821-7802

Ivone Gabriel Ortega- $\mathrm{A}^{2}$

https://orcid.org/0000-0002-4391-9848

J. Franco Rodriguez-Alarcón ${ }^{1,3}$

https://orcid.org/0000-0003-4059-8214

Dennis Arias-Chavez ${ }^{4}$

https://orcid.org/0000-0003-1500-8366

Martin A. Vilela-Estrada ${ }^{5}$

https://orcid.org/0000-0002-1494-952X

Victor Serna-Alarcón ${ }^{5}$

https://orcid.org/0000-0002-9803-6217

Christian R. Mejia ${ }^{6 *}$

https://orcid.org/0000-0002-5940-7281

Aceptado: 06/072021

\section{RESUMEN}

Ahora que contamos con las vacunas contra la COVID-19 para mujeres embarazadas, es importante conocer su percepción y su intención de vacunarse. El objetivo fue determinar la actitud de las mujeres embarazadas hacia la vacunación contra la COVID-19 en el Perú y los factores asociados a esta. Estudio del tipo transversal analítico, esto se realizó en los 24 departamentos peruanos durante los meses de diciembre del 2020 y enero del 2021. El 50\% de las encuestadas aún no decidía si se vacunaría, el 36\% mencionaban que sí se vacunarían, el 8\% mencionó que por ninguna circunstancia lo haría y el 6\% lo haría solo si la obligaban. En el análisis multivariado se encontró que no se vacunarían debido a que no confían en el sistema de salud (valor $\mathrm{p}=0,002$; siendo más las gestantes que desconfiaban del sector salud), en cambio, en los motivos por los que si se vacunarían, se encontró que esta vacunación mejoraría la salud de su familia o seres queridos (valor $\mathrm{p}=0,005$; fueron menos las gestantes que se vacunarían por esta razón). Se concluye que el porcentaje de mujeres embarazadas que sí se vacunarían es bajo, siendo la principal razón por la mejora de la salud de su familia, mientras que la desconfianza en el sistema de salud, el desconocimiento de la elaboración de las vacunas y el no considerarse una población de riesgo fueron los principales factores asociados a la resistencia a la vacunación.

Palabras clave: COVID-19, gestación, vacunación, Perú

\section{ABSTRACT}

Now that COVID-19 vaccines are available for pregnant women, it is important to know their perception and intention to be vaccinated. The objective was to determine the attitude of pregnant women towards vaccination against COVID-19 in Peru and the factors associated with it. This cross-sectional analytical study was conducted in the 24 Peruvian departments during the months of December 2020 and January 2021 . Fifty percent of the respondents had not yet decided whether they would be vaccinated, $36 \%$ said they would be vaccinated, $8 \%$ said they would not be vaccinated under any circumstances, and $6 \%$ would only be vaccinated if they were forced to do so. In the multivariate analysis, it was found that they would not be vaccinated because they did not trust the health system ( $p$-value=0.002; more pregnant women did not trust the health sector), on the other hand, in the reasons why they would be vaccinated, it was found that this vaccination would improve the health of their family or loved ones ( $p$ value $=0.005$; fewer pregnant women would be vaccinated for this reason). It is concluded that the percentage of pregnant women who would get vaccinated is low, being the main reason for the improvement of the health of your family, while distrust in the health system, lack of knowledge of the development of vaccines and not considering themselves a population at risk were the main factors associated with resistance to vaccination.

Key words: COVID-19, pregnancy, vaccination, Peru.

1. Asociación Médica de Investigación y Servicios en Salud. Lima, Perú. 2. Universidad Continental. Huancayo, Perú.

3. Facultad de Medicina Humana "Manuel Huamán Guerrero”. Universidad Ricardo Palma. Lima, Perú.

4. Universidad Continental. Arequipa, Perú.

5. Escuela de Medicina Humana, Universidad Privada Antenor Orrego. Trujillo, Perú. 6. Centro de Investigación en Medicina Traslacional. Universidad Norbert Wiener. Lima, Perú. 


\section{Introducción}

La pandemia de la COVID-19 ha tenido múltiples repercusiones a nivel mundial, siendo una de las principales el colapso de los sistemas de salud, sobre todo en Latinoamérica, donde algunos países ya enfrentaban una crisis sanitaria que se sumaba a las desigualdades sociales (Cabezas, 2021). Ante esta situación, las vacunas parecen ser la solución, ya que, si bien no evitan que contraigas la enfermedad al 100\%, sí ayudan a que las personas que se la apliquen no tengan una presentación severa de la enfermedad, disminuyendo de esta manera las tasas de mortalidad, la necesidad de intubación e ingreso a la unidades de cuidados intensivos (UCI) (Chemaitelly et al., 2021; Hall et al., 2021). Esto resulta importante, pues en la actualidad ya contamos con una decena de vacunas a nivel mundial, y muchas ya han empezado a utilizarse en varios países desde diciembre del año pasado. Esto ha generado que algunos países como Israel, Inglaterra, Estados Unidos, entre otros, hayan llegado a alcanzar buenos niveles de inmunidad en su población; inclusive en estos son donde primero se están levantando muchas de las restricciones sociales (Baños, 2021; BBC, 2021). Sin embargo, todavía hay muchos países que no cuentan con porcentajes idóneos de tasas vacunación, cómo es el caso de Perú, y a esto se suma que todavía hay algunos grupos poblacionales que, por diversos motivos, se muestran reacios a vacunarse, o que aún tienen dudas acerca de si se vacunarían o no (Herrera-Añazco et al., 2021).

Uno de estos grupos son las gestantes, ya que, estas no han sido incluidas en la mayoría de ensayos clínicos a nivel mundial; sin embargo, debido a la alteración en la respuesta inmunitaria que tiene lugar durante el embarazo, las gestantes tienen mayor riesgo de enfermarse gravemente por las infecciones virales, como la COVID-19 (Chen et al., 2020). Es por eso que algunas vacunas ya están siendo probadas en esta población, sobre todo las vacunas que se basan en el ARNm, mostrando buenos resultados y una tasa de eventos adversos similar al de las mujeres no embarazadas, siendo las complicaciones obstétricas extremadamente raras (Bookstein Peretz et al., 2021). No obstante, la información acerca de estas vacunas y sus efectos sobre la gestación son limitados, pero todo parece indicar que el riesgo de la COVID-19 materna es mucho más grave que los riesgos de la vacunación (Brillo et al., 2021). Por eso resulta importante, ahora que contamos con la vacuna para estas mujeres, conocer su percepción y su intención de vacunarse llegado el momento; sin embargo, en el Perú aún no existen muchos estudios que evalúen el porcentaje de aceptación para vacunarse contra el SARS-CoV-2 en mujeres gestantes y no gestantes, por todo esto es que el objetivo de la investigación fue determinar la actitud de las mujeres embarazadas hacia la vacunación contra la COVID-19 en el Perú y los factores asociados a esta.

\section{Materiales y Métodos}

La investigación tuvo un diseño del tipo transversal analítico, esto se realizó en los 24 departamentos peruanos durante los meses de diciembre del 2020 y enero del 2021. Se obtuvo una muestra por conveniencia de las mujeres mayores de 18 años que aceptaron ser parte del estudio y respondieron acerca de su posibilidad de vacunarse. Se excluyó a aquellas que dieron datos incompletos o incoherentes (exclusión menor al 3\%). Para este estudio se calculó la potencia estadística, que significa el cruce de las variables acerca si se vacunará contra las otras. En donde cada uno de las cruces fue igual o mayor al $80 \%$ de la potencia estadística; lo que significa que es adecuada para nivel de investigación.

La variable principal se obtuvo mediante la manifestación de la intención de vacunarse contra el coronavirus, en donde se preguntó directamente si lo harían o no. Además, se obtuvo 7 preguntas de los motivos de por qué si y no lo harían. Esto obtenido de un cuestionario validado en el Perú para este fin, que ha demostrado una validez interna y confiabilidad para ver los factores más importantes de los motivos de la vacunación (Mejia et al., 2021).

También se consignó la edad de las encuestadas, el departamento donde residía en el momento de la encuesta, si es que padecían alguna enfermedad importante (diabetes, enfermedad cardiovascular, VIH, entre otros), también se midió su grado de instrucción (secundaria o menos, estudios técnicos, superiores o postgrado) y se indagó si había padecido de COVID-19 o no en los meses previos.

La investigación primaria se realizó con el objetivo de conocer los motivos de la vacunación en una población mayor, sin embargo, se realizó esta investigación de análisis secundario de datos para ver una subpoblación específica que es de interés de la salud pública (mujeres gestantes y no gestantes). Para la investigación principal se obtuvo la aprobación del Comité de Ética de la Universidad Norbert Wiener (expediente $\mathrm{N}^{\circ}$ 306-2020) y luego se realizó una encuesta virtual a través del SurveyMonkey, en donde se indagó acerca la posibilidad de vacunarse y otras variables importantes para el estudio. Una vez que se obtuvo la cantidad total de respuestas se realizó la exportación de las respuestas al programa Microsoft Excel, aquí se realizó el control de la calidad de los datos, primero realizado en el programa Microsoft Excel. Posteriormente se exportó la información a una hoja del programa Stata versión 11,1 (versión adquirida por el estadístico del grupo). 
La primera etapa de análisis de datos consistió en la obtención de las frecuencias y porcentajes de las variables categóricas, así como, de las medidas de tendencias central y de dispersión de las variables cuantitativas. Luego de esto se pasó a realizar la estadística bivariada y multivariada, esto con la ayuda modelos lineales generalizados (familia Poisson, función de log, modelos para varianzas robustas y ajustando por el departamento residencia). Con todo esto se obtuvo las razones de prevalencia crudas (análisis bivariado) y las razones de prevalencia ajustadas (análisis multivariado), así como, los intervalos de confianza (para ambos modelos) y valores p (para ambos modelos). En toda esta estadística analítica se obtuvo un nivel de confianza del $95 \%$ y se consideró como estadísticamente significativo al valor de 0,05 .

\section{Resultados}

De las 5863 encuestadas, se encontró que hubo diferencias de estar actualmente en gestación según el estado civil ( $\mathrm{p}<0,001)$, la edad de la madre $(\mathrm{p}<0,001)$ y el grado de instrucción que poseían $(\mathrm{p}<0,001)$; sin embargo, no hubo diferencias según el si se vacunaría contra el COVID-19 (p=0,198) (Tabla 1).

Tabla 1. Características de las encuestadas en Perú previo a la llegada de la vacuna contra la COVID-19

\begin{tabular}{lccc}
\hline \multicolumn{1}{c}{ Variable } & Embarazada n (\%) & \multirow{2}{*}{ Valor p } \\
\cline { 2 - 3 } Estado civil & No & Si & \\
$\quad$ Soltera & $4457(97,6 \%)$ & $108(2,4 \%)$ & \\
Casada & $550(80,1 \%)$ & $137(19,9 \%)$ & \\
Conviviente & $302(78,0 \%)$ & $85(22,0 \%)$ & \\
Divorciada & $92(86,0 \%)$ & $15(14,0 \%)$ & \\
Viuda & $92(87,0 \%)$ & $15(14,0 \%)$ & \\
Otro & $40(90,9 \%)$ & $4(9,1 \%)$ & $<0,001$ \\
Edad & & & \\
18-35 años & $4558(95,9 \%)$ & $195(4,1 \%)$ & \\
36 a más años & $949(85,5 \%)$ & $161(14,5 \%)$ & $<0,001$ \\
Instrucción & $878(96,7 \%)$ & $30(3,3 \%)$ & \\
Secundaria o menos & $470(89,0 \%)$ & $58(11,0 \%)$ & \\
Técnico & $2659(97,3 \%)$ & $73(2,7 \%)$ & \\
Superior incompleto & $1138(89,5 \%)$ & $134(10,5 \%)$ & \\
Superior completo & $362(85,6 \%)$ & $61(14,4 \%)$ & $<0,001$ \\
Posgrado & & & \\
Se vacunaría contra el COVID-19 & $387(93,7 \%)$ & $26(6,3 \%)$ & \\
No, por ninguna circunstancia & $2216(94,6 \%)$ & $127(5,4 \%)$ & \\
Sí & $336(94,1 \%)$ & $21(5,9 \%)$ & \multirow{2}{*}{0,198} \\
Sí, pero solo si me obligan & $2361(93,1 \%)$ & $175(6,9 \%)$ & \\
Lo pensaré & & \\
\hline$\quad$ Los valores p se obtuvieron con la prueba estadística del chi cuadrado. &
\end{tabular}

El $50 \%$ de las encuestadas aún no decidía si se vacunaría, seguidas del 36\%, que mencionaban que si se vacunarían. Es importante mencionar que el $8 \%$ mencionó que por ninguna circunstancia lo haría y el $6 \%$ lo haría solo si la obligan (Figura 1).

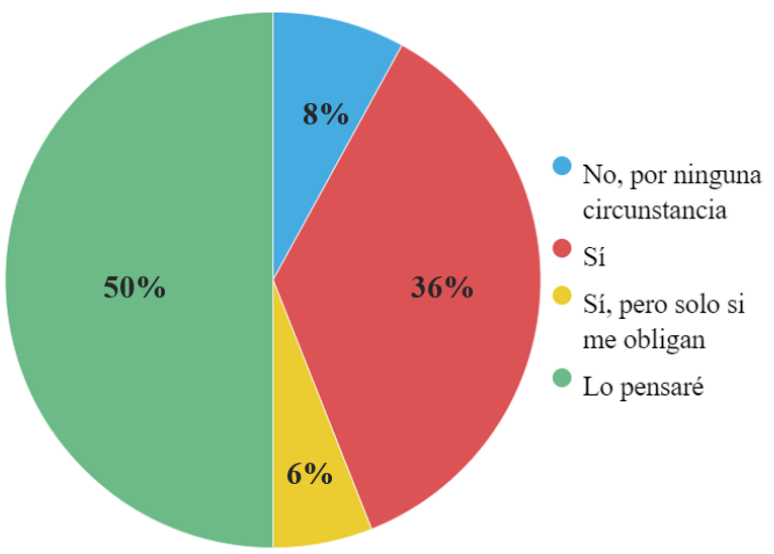

Figura 1. Porcentajes de aceptación de la vacuna contra la COVID-19 en mujeres gestantes peruanas 
Cuando se realizó el análisis bivariado de las razones por las que se vacunarían o no lo harían según el estar gestando, se encontró que hubo diferencia estadísticamente significativa en la premisa que mencionaba que no se vacunaría debido a que no confía en el sistema de salud (valor $\mathrm{p}=0,002$; siendo más las gestantes que desconfiaban del sector salud), en cambio, en los motivos por los que si se vacunarían, hubo diferencia en el saber que esta vacunación mejoraría la salud de su familia o seres queridos (valor $\mathrm{p}=0,005$; fueron menos las gestantes que se vacunarían por esta razón) (Tabla 2).

Tabla 2. Análisis bivariado de las razones por las que se vacunarían según el estar gestando y previo a la llegada de la vacuna contra la COVID-19

\begin{tabular}{|c|c|c|c|}
\hline \multirow{2}{*}{ Muy de acuerdo o de acuerdo con que no me la pondré, porque: } & \multicolumn{2}{|c|}{ Está embarazada } & \multirow[t]{2}{*}{ Valor p } \\
\hline & No & $\mathbf{S i}$ & \\
\hline Pienso que me van a insertar chips/transistores que me harán control mental. & $426(92,4 \%)$ & $38(7,6 \%)$ & 0,135 \\
\hline Pienso que es parte del plan de una gran empresa que creó la enfermedad. & $1105(93,1 \%)$ & $82(6,9 \%)$ & 0,177 \\
\hline $\begin{array}{l}\text { Pienso que si viene de un país comunista (como Rusia), tendré efectos en el } \\
\text { pensamiento comunista }\end{array}$ & $331(94,0 \%)$ & $21(6,0 \%)$ & 0,932 \\
\hline $\begin{array}{l}\text { Pienso que la enfermedad es un invento de la Organización Mundial de la } \\
\text { Salud (OMS) u otras instituciones. }\end{array}$ & $788(93,4 \%)$ & $56(6,6 \%)$ & 0,459 \\
\hline Pienso que la enfermedad COVID-19 no existe, es un invento. & $316(92,7 \%)$ & $25(7,3 \%)$ & 0,316 \\
\hline Una vida saludable es suficiente para combatir las enfermedades. & $1858(93,3 \%)$ & $133(6,7 \%)$ & 0,162 \\
\hline $\begin{array}{l}\text { No confío en mi sistema de salud (incluido el personal de salud). } \\
\text { Muy de acuerdo o de acuerdo con que sí me vacunaría, porque: }\end{array}$ & $1695(92,5 \%)$ & $138(7,5 \%)$ & 0,002 \\
\hline Porque quiero regresar a mi vida antes de la pandemia. & $3539(94,2 \%)$ & $220(5,8 \%)$ & 0,347 \\
\hline Porque mejoraría la salud de mi familia o seres queridos. & $3899(94,5 \%)$ & $227(5,5 \%)$ & 0,005 \\
\hline Creo que mejoraría la salud de la comunidad/población. & $3845(94,2 \%)$ & $239(5,8 \%)$ & 0,285 \\
\hline Porque no quiero seguir usando equipos de protección personal. & $2595(93,6 \%)$ & $177(6,4 \%)$ & 0,341 \\
\hline
\end{tabular}

Al indagarse otras de otras razones por las que no se vacunaría, se encontró que hubo diferencias según el que las mujeres desconocían del proceso de elaboración o de cómo habían hecho las vacunas (valor p<0,001; fue mayor el porcentaje de las gestantes que no se vacunarían por esta razón), así como, por el hecho de que no consideran que pertenezcan a un grupo de riesgo (valor $\mathrm{p}=0,030$; fue menor el porcentaje de las gestantes que no se vacunarían por esta razón) (Tabla 3).

Tabla 3. Análisis bivariado de otras razones por las que se vacunarían según el estar gestando y previo a la llegada de la vacuna contra el COVID-19

\begin{tabular}{|c|c|c|c|}
\hline \multirow{2}{*}{ Muy de acuerdo o de acuerdo con que no me la pondré, porque: } & \multicolumn{2}{|c|}{ Está embarazada } & \multirow[t]{2}{*}{ Valor $\mathbf{p}$} \\
\hline & No & $\mathbf{S i}$ & \\
\hline Ya me infecté de COVID-19 y pienso que no es necesario. & $396(92,3 \%)$ & $33(7,7 \%)$ & 0,144 \\
\hline Creo que la pandemia ya se está acabando. & $511(92,9 \%)$ & $39(7,1 \%)$ & 0,293 \\
\hline Desconozco de que está elaborada o cómo la han hecho. & $2058(92,5 \%)$ & $166(7,5 \%)$ & $<0,001$ \\
\hline Puede generarme efectos secundarios o adversos. & $2564(93,5 \%)$ & $178(6,5 \%)$ & 0,207 \\
\hline Creo que quieren experimentar con nosotros. & $1415(93,0 \%)$ & $107(7,0 \%)$ & 0,069 \\
\hline Considero que no pertenezco a un grupo de riesgo. & $1530(95,0 \%)$ & $80(5,0 \%)$ & 0,030 \\
\hline \multicolumn{4}{|l|}{ Muy de acuerdo o de acuerdo con que sí me vacunaría, porque: } \\
\hline Solo si es requisito para el trabajo o para el estudio. & $1733(94,4 \%)$ & $103(5,6 \%)$ & 0,317 \\
\hline Si la vacunación fuera a domicilio. & $2786(93,9 \%)$ & $182(6,1 \%)$ & 0,845 \\
\hline
\end{tabular}

Los valores p se obtuvieron con la prueba estadística del chi cuadrado.

\section{Discusión}

En este estudio se encontró qué la mitad de las mujeres gestantes encuestadas aún tenían dudas acerca de la vacunación contra la COVID-19, y solo un $36 \%$ estaba segura de que sí se vacunaría. Estos resultados son similares a los encontrados en otro estudio, donde solo el $41 \%$ de las mujeres gestantes estaba dispuesta a recibir la vacuna llegado el momento, siendo las mujeres afroamericanas e hispanas quienes mostraban menor predisposición a vacunarse (Battarbee et al., 2021). Estos bajos porcentajes podrían deberse a la escasa información que se tiene en la actualidad acerca de los efectos adversos y la seguridad de la vacuna en las mujeres gestantes y en periodo de lactancia, reportándose en un estudio previo que la mayoría de las mujeres embarazadas $(70,0 \%)$ y que daban de lactar $(83,7 \%)$ estarían dispuestas a vacunarse solo si se publicaran más datos acerca de la seguridad de la vacuna durante el embarazo y la lactancia (Jayagobi et al., 2021). Esto evidencia la necesidad de incluir a este grupo poblacional en los estudios para el desarrollo de vacunas y medicamentos contra la COVID-19, pues si bien la Administración de Alimentos y Medicamentos de los Estados Unidos (FDA, por sus siglas en inglés) dejó abierta la opción de que las mujeres 
embarazadas y lactantes recibieran la vacuna, resulta complicado que la población y los propios profesionales de la salud tomen decisiones sin la evidencia suficiente.

No obstante, es importante mencionar que en algunos estudios se ha encontrado que las mujeres embarazadas tenían menos predisposición a vacunarse que las mujeres no gestantes (Skirrow et al., 2021; Skjefte et al., 2021; Sutton et al., 2021). Esto probablemente se deba a que las mujeres gestantes no solo se preocupan de los efectos adversos de las vacunas en ellas, sino de la seguridad y el bienestar del nonato; siendo esto uno de los principales motivos por los que las mujeres gestantes tomarían la decisión de vacunarse o no (Geoghegan et al., 2021). Hasta el momento, los estudios que se han realizado en esta población no han mostrado que la vacuna pueda generar daño al feto (Male, 2021), no obstante, las complicaciones fetales de la COVID-19 durante el embarazo incluyen aborto espontáneo (2\%), restricción del crecimiento intrauterino (RCIU; 10\%) y parto prematuro (39\%), entre otros (Dashraath et al., 2020). Frente a esta situación, si bien no existen muchos datos acerca de los efectos adversos de la vacuna en el feto, el riesgo de la infección es mayor a los posibles efectos adversos, por lo cual, es importante que se difunda esta información hacia las mujeres gestantes, pues este es un factor que podría influir en su decisión de vacunación.

Un hallazgo importante en este estudio fue que la percepción de mejorar la salud de su familia o seres queridos era una de las principales razones por la cual las mujeres se vacunarían, siendo menos las gestantes quienes se vacunarían por esta razón. Estudios previos han reportado que la vacuna contra la COVID-19 no solo protege a la persona que se la coloca, sino que también ayuda a que la transmisión del virus disminuya 40-50\% entre los miembros de su hogar (Harris et al., 2021; Salo et al., 2021). Por otro lado, en el caso de las embarazadas, la vacunación también podría proteger al feto, pues algunos estudios encontraron que las mujeres que recibieron vacunas de tipo ARNm contra el COVID-19 durante el embarazo (mayormente durante su tercer trimestre) les han transmitido anticuerpos a los fetos, lo que podría ayudar a protegerlos luego de nacer (Burd et al., 2021; Chavan et al., 2021). Entonces es importante que tanto las mujeres embarazadas como las no embarazadas consideren vacunarse, pues de esta manera se protegen ellas, pero también protegen a sus seres queridos. Por lo que, esta razón debe seguir investigándose, ya que, los resultados nos indican que podría haber muchas más razones detrás de la percepción de vacunarse como sentido de protección familiar.

Por otro lado, una de las razones por las que no se vacunarían fue porque desconfiaban del sistema de salud, siendo esta desconfianza mayor en las gestantes. Otros estudios han reportado resultados similares, donde la confianza en las instituciones de salud y la información recibida por estas fueron importantes predictores para que las mujeres gestantes acepten la vacunación contra la COVID-19 (Levy et al., 2021; Skirrow et al., 2021; Skjefte et al., 2021). Este comportamiento también se ha visto en la población en general, en un estudio realizado en 19 países se encontró que, si una persona tenía mayor confianza en su gobierno, entonces era más probable que tuviera una actitud positiva hacia la vacunación, o también si algún miembro del personal de salud se lo recomendaba (Lazarus et al., 2021). Por lo cual, una comunicación clara por parte de las instituciones de salud y del gobierno es clave para que esta campaña de vacunación tenga éxito.

Las encuestadas también mencionaron que una de las razones por la que no se vacunarían era porque desconocían el proceso de elaboración de las vacunas, siendo más las que gestantes quienes no se vacunarían por esta razón. Resultados similares se han visto en otros estudios, donde las gestantes manifestaron mayor desconfianza en las vacunas, en comparación con las no gestantes (Skirrow et al., 2021), siendo además uno de los motivos más frecuentes de rechazo a la vacunación la falta de datos acerca de su seguridad (Goncu Ayhan et al., 2021). Este motivo podría estar relacionado a las noticias falsas que se difundieron desde el inicio de la pandemia, pues un estudio realizado entre diciembre de 2019 y noviembre de 2020 encontró que, el $91 \%$ de la información difundida en las redes sociales y en internet eran rumores, y de estos, el 36\% guardaban relación con el desarrollo, la disponibilidad y el acceso a las vacunas contra la COVID-19, además el $83 \%$ de la información era falsa (Islam et al., 2021). La mayoría de estos rumores y noticias falsas ya han sido desmentidas (CDC, 2021; Hotez et al., 2021). Sin embargo, estos resultados evidencian que probablemente se tenga que intensificar la lucha contra las "fake news" y mejorar el acceso a información de calidad en la población.

Por último, otra de las razones por las que no se vacunarían es por no considerar que pertenezcan a un grupo de riesgo. Esto guarda relación con lo mencionado por Tao et al., (2021) quienes encontraron que existe una asociación entre la tasa de aceptación de la vacunación y la percepción de susceptibilidad que tienen las mujeres gestantes. No obstante, es importante mencionar que en nuestro estudio fue menor el porcentaje de las gestantes quienes no se vacunarían por esta razón. Esto es importante, pues durante el embarazo se producen varios cambios fisiológicos y hormonales que la predisponen a la infección de algunos patógenos respiratorios (Chen et al., 2020), tal y como se ha visto en pandemias previas, como el Síndrome Respiratorio Agudo Severo (SARS) y el Síndrome Respiratorio de Oriente Medio (MERS) (Babarinsa et al., 2021). Por esto es importante no solo que se priorice a este grupo de mujeres en la vacunación, sino que se realicen campañas de difusión de la información y concientización para que las gestantes se muestren más dispuestas a vacunarse. 
A pesar de tener casi 6 mil encuestadas a nivel Perú, este estudio tiene la limitación de que no se pueda extrapolar a la totalidad de mujeres o a algún departamento en específico donde se tomó la encuesta; esto debido a que no se realizó un muestreo de tipo aleatorio; sin embargo, los resultados todavía permiten tener asociaciones estadísticas importantes que muestran los factores que influyen en la decisión de las mujeres gestantes de vacunarse o no. Los resultados de este estudio pueden tomarse como exploratorios o primarios, sentando una base para futuras investigaciones que consideren una población mayor, con un muestreo aleatorio, con mayor cantidad de variables que tratan de explicar la percepción de vacunarse y con diseños de investigación de tipo causa-efecto.

Por todo lo mencionado se concluye que el porcentaje de mujeres que sí se vacunarían es bajo, además la mitad de las encuestadas aún no decidía si se vacunaría o no; además se encontró que la principal razón por la que las mujeres se vacunarían sería por mejorar la salud de su familia, mientras que la desconfianza en el sistema de salud, el desconocimiento de la elaboración de las vacunas y el no considerarse una población de riesgo fueron los principales factores asociados a no querer vacunarse. Se sugiere que se realicen estudios que midan la evolución de la intención de vacunarse, ya que, nuestros resultados muestran esta realidad en las primeras semanas del 1er año de disponibilidad de las vacunas (así que es básicamente una investigación previa a que lleguen), por lo que, se deben tomar más mediciones en el paso del tiempo y la disponibilidad de los fármacos.

\section{Conflicto de intereses}

Los autores declaran no tener conflicto de interés.

\section{Agradecimientos}

A la red de investigación COVID-19-GIS-Peru, por su respaldo para el encuestado; así como, a la Universidad Norbert Wiener, por el financiamiento para la realización de la investigación primaria, que permitió la recolección de data.

\section{Referencias}

Babarinsa, I. A., Okunoye, G. O., \& Odukoya, O. (2021). Severe Acute Respiratory Syndrome Coronavirus (SARS-CoV-1) and Middle East Respiratory Syndrome Coronavirus (MERS-CoV) infections in pregnancy - An overview. European Journal of Obstetrics and Gynecology and Reproductive Biology, 263(1), 171-175. https://doi.org/10.1016/j.ejogrb.2021.06.020

Baños, J. (2021). Israel levanta el confinamiento gracias a su eficacia al vacunar. La Vanguardia. Disponivble en: https://www.lavanguardia.com/internacional/20210221/6257361/israel-vacunacionefectividad-pandemia-confinamiento-impacto.html (Acceso marzo 2021)

Battarbee, A. N., Stockwell, M. S., Varner, M., Newes-Adeyi, G., Daugherty, M., Gyamfi-Bannerman, C., Tita, A., Vorwaller, K., Vargas, C., Subramaniam, A., Reichle, L., Galang, R., Powers, E., LuccaSusana, M., Parks, M., Chen, T. J., Razzaghi, H., \& Dawood, F. S. (2021). Attitudes toward COVID19 illness and COVID-19 vaccination among pregnant women: A cross-sectional multicenter study during August-December 2020. MedRxiv, Preprint. https://doi.org/10.1101/2021.03.26.21254402

BBC. (2021). Inglaterra levanta la mayoría de las restricciones para combatir el contagio de coronavirus, en medio de llamados a la cautela. BBC News Mundo. Disponivble en: https://www.bbc.com/mundo/noticias-internacional-57883427 (Acceso marzo 2021)

Bookstein Peretz, S., Regev, N., Novick, L., Nachshol, M., Goffer, E., Ben-David, A., Asraf, K., Doolman, R., Sapir, E., Yochay, G. R., \& Yinon, Y. (2021). Short-term outcome of pregnant women vaccinated by BNT162b2 mRNA COVID-19 vaccine. Ultrasound in Obstetrics \& Gynecology, Preprint. https://doi.org/10.1002/uog.23729

Brillo, E., Tosto, V., Gerli, S., \& Buonomo, E. (2021). COVID-19 vaccination in pregnancy and postpartum. The Journal of Maternal-Fetal \& Neonatal Medicine, $0(0), \quad 1-20$. https://doi.org/10.1080/14767058.2021.1920916

Burd, I., Kino, T., \& Segars, J. (2021). The Israeli study of Pfizer BNT162b2 vaccine in pregnancy: Considering maternal and neonatal benefits. The Journal of Clinical Investigation, 131(13), e150790. https://doi.org/10.1172/JCI150790 
Cabezas, C. (2021). Pandemia de la COVID-19: Tormentas y retos. Revista Peruana de Medicina Experimental y Salud Pública, 37(4), 603-604. https://doi.org/10.17843/rpmesp.2020.374.6866

CDC. (2021, julio 7). Datos sobre las vacunas contra el COVID-19. Centers for Disease Control and Prevention. Disponible en: https://espanol.cdc.gov/coronavirus/2019-ncov/vaccines/facts.html (Acceso marzo 2021)

Chavan, M., Qureshi, H., Karnati, S., \& Kollikonda, S. (2021). COVID-19 Vaccination in Pregnancy: The Benefits Outweigh the Risks. Journal of Obstetrics and Gynaecology Canada, 43(7), 814-816. https://doi.org/10.1016/j.jogc.2021.03.010

Chemaitelly, H., Yassine, H. M., Benslimane, F. M., Al Khatib, H. A., Tang, P., Hasan, M. R., Malek, J. A., Coyle, P., Ayoub, H. H., Al Kanaani, Z., Al Kuwari, E., Jeremijenko, A., Kaleeckal, A. H., Latif, A. N., Shaik, R. M., Abdul Rahim, H. F., Nasrallah, G. K., Al Kuwari, M. G., Al Romaihi, H. E., ... AbuRaddad, L. J. (2021). MRNA-1273 COVID-19 vaccine effectiveness against the B.1.1.7 and B.1.351 variants and severe COVID-19 disease in Qatar. Nature Medicine, 1-8. https://doi.org/10.1038/s41591-021-01446-y

Chen, M., Zeng, J., Liu, X., Sun, G., Gao, Y., Liao, J., Yu, J., Luo, X., \& Qi, H. (2020). Changes in physiology and immune system during pregnancy and coronavirus infection: A review. European Journal of Obstetrics \& Gynecology and Reproductive Biology, 255(1), 124-128. https://doi.org/10.1016/j.ejogrb.2020.10.035

Dashraath, P., Wong, J. L. J., Lim, M. X. K., Lim, L. M., Li, S., Biswas, A., Choolani, M., Mattar, C., \& Su, L. L. (2020). Coronavirus disease 2019 (COVID-19) pandemic and pregnancy. American Journal of Obstetrics and Gynecology, 222(6), 521-531. https://doi.org/10.1016/j.ajog.2020.03.021

Geoghegan, S., Stephens, L. C., Feemster, K. A., Drew, R. J., Eogan, M., \& Butler, K. M. (2021). “This choice does not just affect me." Attitudes of pregnant women toward COVID-19 vaccines: A mixedmethods study. Human Vaccines \& Immunotherapeutics, 1-6. https://doi.org/10.1080/21645515.2021.1924018

Goncu Ayhan, S., Oluklu, D., Atalay, A., Beser, D. M., Tanacan, A., Tekin, O. M., \& Sahin, D. (2021). COVID-19 vaccine acceptance in pregnant women. International Journal of Gynecology \& Obstetrics, 154(2), 291-296. https://doi.org/10.1002/ijgo.13713

Hall, V. J., Foulkes, S., Saei, A., Andrews, N., Oguti, B., Charlett, A., Wellington, E., Stowe, J., Gillson, N., Atti, A., Islam, J., Karagiannis, I., Munro, K., Khawam, J., Chand, M. A., Brown, C. S., Ramsay, M., Lopez-Bernal, J., Hopkins, S., ... Heeney, J. (2021). COVID-19 vaccine coverage in health-care workers in England and effectiveness of BNT162b2 mRNA vaccine against infection (SIREN): A prospective, multicentre, cohort study. The Lancet, 397(10286), 1725-1735. https://doi.org/10.1016/S0140-6736(21)00790-X

Harris, R. J., Hall, J. A., Zaidi, A., Andrews, N. J., Dunbar, J. K., \& Dabrera, G. (2021). Effect of Vaccination on Household Transmission of SARS-CoV-2 in England. N Engl J Med, Preprint.

Herrera-Añazco, P., Uyen-Cateriano, A., Urrunaga-Pastor, D., Bendezu-Quispe, G., Toro-Huamanchumo, C. J., Rodriguez-Morales, A. J., Hernandez, A. V., \& Benites-Zapata, V. A. (2021). Prevalence and factors associated with the intention of vaccination against COVID-19 in Peru. Prevalence and factors associated with the intention of vaccination against COVID-19 in Peru, Preprint.

Hotez, P., Batista, C., Ergonul, O., Figueroa, J. P., Gilbert, S., Gursel, M., Hassanain, M., Kang, G., Kim, J. H., Lall, B., Larson, H., Naniche, D., Sheahan, T., Shoham, S., Wilder-Smith, A., Strub-Wourgaft, N., Yadav, P., \& Bottazzi, M. E. (2021). Correcting COVID-19 vaccine misinformation: Lancet Commission on COVID-19 Vaccines and Therapeutics Task Force Members*. EClinicalMedicine, 33(1), 1-3. https://doi.org/10.1016/j.eclinm.2021.100780

Islam, S., Mostofa Kamal, A.-H., Kabir, A., Southern, D. L., Khan, S. H., Hasan, S. M. M., Sarkar, T., Sharmin, S., Das, S., Roy, T., Harun, M. G. D., Chughtai, A. A., Homaira, N., \& Seale, H. (2021). COVID-19 vaccine rumors and conspiracy theories: The need for cognitive inoculation against 
misinformation to improve vaccine adherence. PLOS ONE, 16(5), e0251605. https://doi.org/10.1371/journal.pone.0251605

Jayagobi, P. A., Ong, C., Thai, Y. K., Lim, C. C., Jiun, S. M., Koon, K. L., Wai, K. C., Chan, J. K., Mathur, M., \& Chien, C. M. (2021). Perceptions and acceptance of COVID-19 vaccine among pregnant and lactating women in Singapore: A cross-sectional study. MedRxiv, Preprint. https://doi.org/10.1101/2021.06.29.21259741

Lazarus, J. V., Ratzan, S. C., Palayew, A., Gostin, L. O., Larson, H. J., Rabin, K., Kimball, S., \& ElMohandes, A. (2021). A global survey of potential acceptance of a COVID-19 vaccine. Nature Medicine, 27(2), 225-228. https://doi.org/10.1038/s41591-020-1124-9

Levy, A. T., Singh, S., Riley, L. E., \& Prabhu, M. (2021). Acceptance of COVID-19 vaccination in pregnancy: A survey study. American Journal of Obstetrics \& Gynecology Mfm, 3(5), 100399. https://doi.org/10.1016/j.ajogmf.2021.100399

Male, V. (2021). Are COVID-19 vaccines safe in pregnancy? Nature Reviews Immunology, 21(4), 200-201. https://doi.org/10.1038/s41577-021-00525-y

Mejia, C. R., Rodriguez-Alarcon, J. F., Ticona, D., Flores-Lovon, K., Paredes Obando, M., Avalos-Reyes, M. S., Ccasa-Valero, L., Carbajal, M., Carranza-Esteban, R. F., Mamani-Benito, O., Rivera-Lozada, O., \& Tovani-Palone, M. R. (2021). Validation of a Scale to Measure the Perception of SARS-CoV-2 Vaccines Acceptance: The VAC-COVID-19 Scale. Eelectron J Gen Med, 18(5), em303. https://doi.org/10.29333/ejgm/11012

Salo, J., Hägg, M., Kortelainen, M., Leino, T., Saxell, T., Siikanen, M., \& Sääksvuori, L. (2021). The indirect effect of mRNA-based Covid-19 vaccination on unvaccinated household members. MedRxiv, Preprint. https://doi.org/10.1101/2021.05.27.21257896

Skirrow, H., Barnett, S., Bell, S., Riaposova, L., Mounier-Jack, S., Kampmann, B., \& Holder, B. (2021). Women's views on accepting COVID-19 vaccination during and after pregnancy, and for their babies: A multi-methods study in the UK. MedRxiv, 2021.04.30.21256240. https://doi.org/10.1101/2021.04.30.21256240

Skjefte, M., Ngirbabul, M., Akeju, O., Escudero, D., Hernandez-Diaz, S., Wyszynski, D. F., \& Wu, J. W. (2021). COVID-19 vaccine acceptance among pregnant women and mothers of young children: Results of a survey in 16 countries. European Journal of Epidemiology, 36(2), 197-211. https://doi.org/10.1007/s10654-021-00728-6

Sutton, D., D’Alton, M., Zhang, Y., Kahe, K., Cepin, A., Goffman, D., Staniczenko, A., Yates, H., Burgansky, A., Coletta, J., Williams, Z., \& Gyamfi-Bannerman, C. (2021). COVID-19 vaccine acceptance among pregnant, breastfeeding, and nonpregnant reproductive-aged women. American $\begin{array}{llllll}\text { Journal of Obstetrics \& Gynecology } & \text { MFM, 3(5), }\end{array}$ https://doi.org/10.1016/j.ajogmf.2021.100403

Tao, L., Wang, R., Han, N., Liu, J., Yuan, C., Deng, L., Han, C., Sun, F., Liu, M., \& Liu, J. (2021). Acceptance of a COVID-19 vaccine and associated factors among pregnant women in China: A multicenter cross-sectional study based on health belief model. Human Vaccines \& Immunotherapeutics, Preprint(0), 1-10. https://doi.org/10.1080/21645515.2021.1892432 